\title{
FETAL OUTCOME OF TERM PREGNANCY WITH NON- REACTIVE NON-STRESS TEST AT BIRAT MEDICAL COLLEGE TEACHING HOSPITAL
}

\author{
Amit Deo ${ }^{1^{*}}$, Raj Deb Mahato ${ }^{2}$, Ram Bhakta Subedi ${ }^{3}$, Tarun Pradhan ${ }^{4}$
}

\begin{abstract}
Affiliation
1. Lecturer, Department of Obstetrics and Gynaecology, Birat Medical College and Teaching Hospital, Nepal

2. Assistant Professor, Department of Obstetrics and Gynaecology, Birat Medical College and Teaching Hospital, Nepal

3. Lecturer, Department of Paediatrics. Birat Medical College and Teaching Hospital, Nepal

4. Associate Professor, Department of Obstetrics and Gynaecology, Birat Medical College and Teaching Hospital, Nepal
\end{abstract}

\section{ARTICLE INFO}

Received : 28 May, 2021

Accepted : 08 July, 2021

Published : 04 November 2021

(C) Authors retain copyright and grant the journal right of first publication with the work simultaneously licensed under Creative Commons Attribution License CC - BY 4.0 that allows others to share the work with an acknowledgment of the work's authorship and initial publication in this journal.

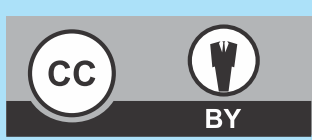

ORA 261

DOI: https://doi.org/10.3126/bjhs.v6i2.40337

* Corresponding Author

Dr. Amit Deo

Lecturer

Department of Obstetrics and Gynaecology

Birat Medical College \& Teaching Hospital, Morang, Nepal Email: dramitdeo368@gmail.com

ORCID: https://orcid.org/0000-0003-0169-2064

\section{Citation}

Amit Deo, Raj Deb Mahato, Ram Bhakta Subedi, Tarun Pradhan. Fetal Outcome of Term Pregnancy with Non-Reactive Non-Stress Test at Birat Medical College Teaching Hospital. BJHS 2021;6(2)15. 1512-1516.

\section{ABSTRACT}

\section{Introduction}

Non-stress test (NST) in pregnancy helps to detect fetuses which are at risk of developing hypoxia. In general, reassuring NST indicates good fetal outcome while nonreassuring NST may result in abnormal perinatal outcome.

\section{Objectives}

The objective of this study is to detect the fetal outcome among term uncomplicated pregnancies with Non-reactive NST in Birat Medical College Teaching Hospital.

\section{Methodology}

A cross-sectional study conducted for 6 months in the department of Obstetrics and Gynaecology at Birat Medical College Teaching Hospital. Singleton pregnancies with longitudinal lie and cephalic presentation between 37 to 42 weeks Period of gestation were included in the study. All participants were advised for a NST as an institutional protocol for 20 minutes at the time of admission. Participants with Non-reactive NST were counselled and planned for emergency LSCS as per hospital protocol. Participants were followed up for intra-operative findings such as colour of liquor and umbilical cord abnormality and outcome of fetus were assessed for APGAR score and need for NICU. The collected data was entered in Microsoft Excel and analyzed by using SPSS version 22 .

\section{Result}

Out of 115 pregnant women, the mean age and standard deviation of $24.93 \pm 4.68$ years. Decelerations was found in $35(30.4 \%)$, Decreased baseline variability was found in $33(28.7 \%)$, Persistent fetal tachycardia was found in $23(20 \%)$ and Decreased baseline fetal heart was found in 24(20.9\%). Intraoperative meconium-stained liquor was found in $39(33.9 \%)$ of patients. All babies born were shown APGAR score $>7$ at 5 minutes. 14(12.2\%) babies were admitted to the Neonatal intensive care unit (NICU). Correlation of admission NST was not found statistically significant with fetal outcome.

\section{Conclusion}

LSCS for abnormal NST does not show adverse fetal outcome.

\section{KEYWORDS}

Fetal Outcome, Non-stress test, Term Pregnancy 


\section{INTRODUCTION}

The maternal mortality rate has significantly decreased in developing countries. Thus, the objectives have shifted toward fetal health. Pregnancy and child birth is a normal physiological phenomenon but has great pathological potential. Most of the pregnancies progress uneventfully but some pregnancies may lead to complications. Present obstetric practice demands care of both mother and her fetus. The fetus is a second patient with a high risk of morbidity and mortality. Fetal surveillance is very important for the delivery of a healthy fetus. The main aim of the current preventive obstetrics practice consists of methods to detect, avoid and treat fetal asphyxia. In the last few decades advances in technology have contributed significantly to improve maternal and perinatal outcomes. During delivery of the fetus through the birth canal is a very stressful condition which can be manifested by the fetus as a stress response in the form of fetal heart rate abnormalities. Some fetuses may have fetal heart rate abnormalities prior to the onset of labor. ${ }^{1}$ To assess the fetal condition during labor is very crucial for the treating obstetrician to minimize perinatal morbidity and mortality. To minimize the unwanted outcome, it is essential to determine the intrauterine fetal conditions which can be achieved by intrapartum fetal monitoring. Intrapartum fetal monitoring gives the idea about fetal condition during labor and identifies fetuses at risk of hypoxic damage so that perinatal outcome can be optimized by appropriate and timely intervention. According to American College of Obstetricians and Gynecologists (ACOG), the aim of antepartum fetal surveillance is to prevent fetal death. ${ }^{2}$ Intrapartum fetal monitoring was traditionally carried out by intermittent auscultation of the fetal heart. Electronic fetal heart rate monitoring (EFM) with Non-Stress Test(NST) is used to record the fetal heart rate (FHR) so as to determine the fetal well-being in order to detect signs of intrapartum hypoxia. Use of intrapartum electronic fetal monitoring with Non-Stress Test has steadily increased over the last three decades in an attempt to decrease the risk of intrapartum fetal morbidity and mortality. ${ }^{3}$ Continuous electronic fetal monitoring may not be possible in low resourced countries like Nepal and hence Non-Stress Test is better alternative. A better understanding of fetal physiology and increasingly advanced technology have changed the attitude towards fetal health. Antepartum assessment of fetal wellbeing is one of the primary tasks of modern obstetric practice. ${ }^{4}$ For predicting pregnancy outcome in uncomplicated pregnancies, we adopted nonstress test (NST). Freeman (1975) and Lee and colleagues introduced the NST. A sign of fetal health describes the fetal heart rate acceleration in response to fetal movement. In fetal asphyxia there is disturbed gas exchange, leading to progressive hypoxemia and hypercapnia with significant metabolic acidosis. ${ }^{5}$ The NST looks for the presence of temporary acceleration of the fetal heart rate at the time of fetal movement that involves the cerebral cortex and is affected by physiologic or pathologic influences on the fetal brain. NST is a continuous recording of fetal heart rate via an ultrasound transducer placed on the mother's abdomen. To assess the fetal well-being during labor and delivery process has been a central component of intrapartum care. The main justification for NST is that the uterine contractions of labor place stress on placental circulation. NST has been able to detect fetal distress with more reliability. ${ }^{6}$ Reactive NST trace reassures both the mother and health care provider of good fetal health. ${ }^{7}$ Abnormal NST is more common in meconium aspiration syndrome. ${ }^{8}$ An abnormal tracing indicates a fetal hypoxia and hence identifies fetal compromise at an early stage to allow an early intervention. Moreover, there is interobserver variation in interpretation of abnormal cardiotocography readings and recommendations for interventions. ${ }^{9}$ The great progress was made in antepartum diagnosis of fetal condition by the introduction of non-stress test (NST). NST registration fetal heartbeats and uterine contractions simultaneously. Fetal heart beats decelerations occur during fetal asphyxia. ${ }^{10}$ Nowadays, almost all pregnant women antenatally monitored with NST, which probably increases the fetal indications of Caesarean sections. ${ }^{10}$ Fetal asphyxia is a condition of disturbed gas exchange, leading to progressive hypoxemia and hypercapnia with significant metabolic acidosis. ${ }^{11}$ Asphyxiated baby may die, recover, manifest hypoxic ischemic encephalopathy (HIE) and later have neurodevelopmental disorders. ${ }^{10}$ The admission nonstress test (NST) is used to indicate the state of oxygenation of the fetus on admission of the mother non- invasively and assess the fetal reserve by recording FHR during the uterine contraction which temporary occlusion of the utero-placental blood supply so non-stress test tracing on admission helps Obstetrician to determine the ability of the fetus to cope with the stress of labour. ${ }^{12}$ APGAR scoring is done at 1 and 5 minutes to assess the health of a new-born baby. A 5-minute APGAR score is of more value for correlation of long-term neurological damage. ${ }^{13}$ Despite NST is associated with increased caesarean section rate, it remains a major method of monitoring high-risk pregnancy. ${ }^{14}$ False positive NST trace means that the record is pathological and child is born, without acidosis; false negative NST trace means that with the normal NST trace and child is born is asphyxiated and depressed child with HIE is born and that will manifest later in neurodevelopmental disorders. This study was conducted with the aim to assess Fetal Outcome at Term Pregnancy with non-reactive NST at Birat Medical College Teaching Hospital.

\section{METHODOLOGY}

A prospective, cross-sectional hospital-based study was conducted for 6 months duration in the department of Obstetrics and Gynecology at Birat Medical College Teaching Hospital (BMCTH). A purposive, total enumeration sampling technique was used to include the participants after permission granted from IRC BMCTH. Informed consent was taken from participants for the study. A singleton uncomplicated healthy pregnancy with longitudinal lie, cephalic presentation at term not in labor with non-reactive NST was included in the study. Complicated pregnancy like hypertensive disorder, GDM, oligohydramnios, multiple gestation, anomalous fetus and patients admitted for 
elective caesarean section were excluded from study. The NST tracings obtained were then categorized as Reactive (Normal), Equivocal (suspicious), \& Non-reactive (Pathological) as per National Institute of Clinical Excellence (NICE) Clinical guideline 2017. Participants fulfilling the inclusion criteria were kept in left lateral position, resuscitation with Dextrose IV fluid and oxygen with face mask were done and then patients were taken for emergency LSCS as per Hospital protocol. Categorical variables such as socio-demographic data and type of non-reactive NST where collected, intraoperative finding such as abnormal liquor colour was noted and fetal outcome was assessed by noting APGAR score at 5 minutes and need of NICU. All the data were entered in Microsoft Excel sheets. Statistical analysis was done for percentage, frequency for categorical variable and chi-square and Pearson's correlation was done for level of significance.

\section{RESULTS}

There were a total of 115 pregnant women with nonreactive NST. The sociodemographic profile of these pregnant women was shown in table 1 . The age of pregnant women is in the range of 18 to 37 years. The mean age and standard deviation of the participants were $24.93 \pm 4.68$ years of which $56.5 \%$ were multigravida, $63.5 \%$ pregnant women were unhooked and $87 \%$ pregnant women from rural areas.

$\begin{array}{ll}\text { Table 1: Sociodemographic profile } & \\ \text { Age (years) } & \mathbf{n}(\%) \\ \leq 20 & 23(20) \\ 21-25 & 48(41.73) \\ 26-30 & 29(25.21) \\ 31-35 & 10(8.69) \\ 35 \text { above } & 5(4.34) \\ \text { Residence } & \\ \text { Rural } & 100(87.00) \\ \text { Urban } & 15(13) \\ & \\ \text { Booking status } & \\ \text { Booked } & \\ \text { Unbooked } & 42(36.5) \\ \text { Gravida } & 73(63.5) \\ \text { Primigravida } & \\ \text { Multigravida } & \end{array}$

Table 2 shows NST finding of the participants. Decelerations were found in $30.4 \%$ of cases, decreased baseline variability was found in $\mathbf{2 8 . 7} \%$ of cases, Persistent fetal tachycardia was found in $20 \%$ of cases and Decreased baseline fetal heart rate in $20.90 \%$ of cases. All the patients with nonreactive NST underwent an emergency caesarean section.
Table 2: Fetal Heart rate pattern in Non-reactive NST ( $n=115$

$\begin{array}{ll}\text { Variable } & \mathbf{n ( \% )} \\ \text { Decelerations } & 35(30.4) \\ \text { Decreased baseline variability } & 33(28.7) \\ \text { Persistent fetal tachycardia } & 23(20.00) \\ \text { Decreased baseline fetal heart rate } & \text { 24(20.90) }\end{array}$

Table 3 shows fetal APGAR score at 5 minutes of birth in Non-reactive NST. $3.5 \%$ of babies had APGAR score $7 / 10$ at 5 minutes of birth, $94.8 \%$ of babies had APGAR score $8 / 10$ at 5 minutes of birth, and $1.7 \%$ of babies had APGAR score $9 / 10$ at 5 minutes of birth.

$\begin{array}{ll}\text { Table 3: APGAR score at } 5 \text { minutes in Non-reactive NST ( } n=115) \\ \text { APGAR score } & \mathbf{n}(\%) \\ 7 / 10 & 4(3.5) \\ 8 / 10 & 109(94.8) \\ 9 / 10 & 2(1.7)\end{array}$

Table 4 shows the cord abnormalities found in Non-reactive NST. $93.9 \%$ of cases no cord abnormalities were found only in $6.1 \%$ of cases cord abnormalities were found.

$\begin{array}{ll}\text { Table 4: Cord abnormalities in Non-reactive NST }(n=115) \\ \text { Cord abnormalities } & \mathbf{n}(\%) \\ \text { Yes } & 7(6.1) \\ \text { No } & 108(93.9)\end{array}$

Table 5 shows NICU admission of baby after delivery of nonreactive NST. only $12.2 \%$ of babies were admitted to NICU after delivery.

$\begin{array}{lc}\text { Table 5: NICU admission in Non-reactive NST }(\mathbf{n}=115) \\ \text { NICU admission } & \mathbf{n}(\%) \\ \text { Yes } & 14(12.2) \\ \text { No } & 101(87.8)\end{array}$

Table 6 shows non-reactive NST and amniotic fluid abnormality. $64.3 \%$ of cases had clear liquor, $33.9 \%$ case had meconium-stained liquor and $1.7 \%$ cases had absent liquor.

\begin{tabular}{|c|c|c|}
\hline \multicolumn{3}{|c|}{$\begin{array}{l}\text { Table 6: Non-reactive NST and amniotic fluid abnormality ( } \mathrm{n}=115 \\
\text { Variables } \\
\mathbf{n}(\%)\end{array}$} \\
\hline Meconium-stained liquor & \multicolumn{2}{|c|}{$39(33.9)$} \\
\hline Absent liquor & \multicolumn{2}{|c|}{$2(1.7)$} \\
\hline Clear liquor & \multicolumn{2}{|c|}{$74(64.3)$} \\
\hline \multicolumn{3}{|c|}{$\begin{array}{l}\text { Table 7: Association between non-reactive NST and NICU } \\
\text { admission ( } n=115) \\
\text { NICU admission }\end{array}$} \\
\hline Yes & $14(12.2)$ & .546 \\
\hline No & $101(87.8$ & \\
\hline
\end{tabular}


Table 8: Association between non-reactive NST and amniotic fluid abnormality ( $n=115)$

$\begin{array}{lll}\text { Variables } & \mathbf{n}(\%) & \text { P value } \\ \text { Meconium-stained liquor } & 39(33.9) & \\ \text { Absent liquor } & 2(1.7) & .290 \\ \text { Clear liquor } & 74(64.3) & \end{array}$

Table 9: Association between non-reactive NST and 5 minutes APGAR score $(n=115)$

\begin{tabular}{lll} 
APGAR score & $\mathbf{n}(\%)$ & P value \\
$7 / 10$ & $4(3.5)$ & .091 \\
$8 / 10$ & $109(94.8)$ & \\
$9 / 10$ & $2(1.7)$ & \\
\hline
\end{tabular}

\begin{tabular}{|c|c|c|}
\hline Cord abnormalities & n (\%) & P value \\
\hline Yes & $7(6.1)$ & .330 \\
\hline No & 108 & (93.9) \\
\hline
\end{tabular}

\section{DISCUSSION}

Non-stress test (NST) has emerged as a modern noninvasive tool for detecting fetal asphyxia in the last decade. NST is one of the reliable methods of fetal monitoring in pregnancy and during labour. ${ }^{15}$ In majority of the hospitals of developed and developing countries, NST is the most commonly used tool for fetal surveillance. NST is superior method for fetal hypoxia detection as it detects, the subtle changes in fetal heart rate which can be missed on intermittent auscultation. ${ }^{16}$ NST reliability as a sole tool in diagnosing fetal distress is questionable and can lead to unnecessary rise in caesarean section rates. This study was conducted to correlate the fetal outcome after non-reactive NST.

In our study, Majority of participants were of age group 2125 years $(41.73 \%)$, followed by $26-30$ years age group (25.21\%). This is similar to study done by Rahman et al in which $42.5 \%$ of the participants were of age group 21-25 years. ${ }^{17}$ This is because of similar study setting. In our study, in Non-reactive NST, $27.9 \%$ patients had meconium-stained liquor, none of newborn had APGAR score less than 7 at 5 minutes and $12.2 \%$ need NICU admission. The finding is

\section{REFERENCES}

1. Hafizur R, Renjhen P, Dutta S, Kar S. Admission cardiotocography: Itsrole in predicting foetal outcome in high-risk obstetric patients. AMJ. 2012; 5(10):522-527. DOI: 10.4066/AMJ.2012.1267

2. Practice bulletin no. 145: antepartum fetal surveillance. Obstet Gynecol. 2014 Jul;124(1):182-192. doi: 10.1097/01. AOG. 0000451759.90082.7b. PMID: 24945455.

3. Gunasena CGA, Jayasundara JMSW. Intra-partum Fetal MonitoringCardotocograph. Sri Lanka J of Obst and Gynae. 2015: 19-26. DOI: http://doi.org/10.4038/sljog.v38i2.7788

4. Meier PR, Makowski EL: The antepartum assessment of fetal wellbeing (Medical Progress). West J Med 1983 May; 138:686-689. PMID:6880185 inconsistent with the study done by Joshi et al (2019) shows $75 \%$ patients had meconium-stained liquor, $6.7 \%$ of the neonates had APGAR less than 7 at 5 minutes and $6.7 \%$ needed NICU admission in Non-reactive NST. ${ }^{18}$ In our study, all patients under gone caesarean section in Non-reactive NST in contrast to study done by Banu et al (2016)shows $80 \%$ patients with Non-reactive NST underwent caesarean section. ${ }^{19}$ In our study caesarean section rates are high in Non-reactive NST because patients and patients party are very much conscious about the baby and don't want to take any risk. In our study admission NST in uncomplicated pregnancies does not show any fetal advantage and increases the caesarean section rate. The finding is consistent with the study done by Bhartiya et al (2016) shows admission NST does not benefit the neonatal outcome in uncomplicated pregnancies and rather results in increased caesarean section rate. ${ }^{20}$

\section{CONCLUSION}

The admission NST is not beneficial for assessing intrapartum fetal hypoxia and increases the need for a caesarean section. So, the caesarean section rate increases. Hence, admission NST does not benefit the neonatal outcome in uncomplicated pregnancies and rather results in increased caesarean section rate. Thus, it is not beneficial as a screening test to detect intrapartum fetal hypoxia in uncomplicated pregnancies.

\section{RECOMMENDATIONS}

A large number of pregnant women based on randomized control trials is required for assessing efficacy of admission NST and fetal outcome in low-risk pregnancies.

\section{LIMITATIONS OF THE STUDY}

NST reporting was done by different doctor so may be observer bias.

\section{ACKNOWLEDGEMENTS}

First and foremost, praises and thanks to women who participate in my study. Thanks to the Obstetrics and Gynaecology department for help and support.

\section{CONFLICT OF INTEREST}

We declare no conflict of interest.

6. Gardosi J. Monitoring technology and the clinical perspective. Bailliere's ClinObstetGynaecol. 1996;10:325-40. DOI: 10.1016/ s09503552(96)80041-1

7. Shiekh SM, Kamruddin A, Setna F, Riaz T. Role of pathological cardiotocography in evaluating fetal well-being. J Coll Physicians Surg Pak. 2006 Jun;16(6):404-7. PMID: 16787617.

8. Ash AK. Managing patients with meconium-stained amniotic fluid. Hosp Med. 2000 Dec;61(12):844-8. doi: 10.12968/ hosp. 2000.61. 12.1482. PMID: 11211584

9. Palomaki O, luukkaala T, Luoto R, Tuimala R. Intrapartum cardiotocography. The dilemma of interpretational variation. J Perinat Med. 2006;34:298-302. DOI: 10.1515/JPM.2006.057 
10. Bogdanovic G, Babovic A, Rizvanovic M, Ljuca D, Grgic G, DjuranovicMilicic J. Cardiotocography in the prognosis of perinatal outcome. Med Arch. 2014;68(2):102-105. doi:10.5455/medarh.2014.68.102105

11. LowJA. Intrapartum fetal asphyxia:Definition, diagnosis and classification. Am J Obstet Gynecol. 1999; 176:(5) 957-959. DOI: 10.1016/s0002-9378(97)70385-5

12. Bhartiya V, Sharma R, Kumar A, Srivastava H. Admission Cardiotocography: A Predictor of Neonatal Outcome. The J of Obs and Gynec of India. 2016; 66(1):321-329. DOI: 10.1007/s13224-016-0912-0

13. Ehrenstein V, Pedersen L, Grijota M, Nielsen GL, Rothman KJ, Sørensen HT. Association of Apgar score at five minutes with long-term neurologic disability and cognitive function in a prevalence study of Danish conscripts. BMC Pregnancy Childbirth. 2009;9:14. Published 2009 Apr 2. doi:10.1186/1471-2393-9-14

14. Bix E, Reiner LM, Klovning A, Oian P. Prognostic value of labour admission test and its effectiveness compared with auscultations only: A systematic review. Brit J ObstetGynaecol. 2005;112:15951604. DOI: $10.1111 / \mathrm{j} .1471-0528.2005 .00766 . x$

15. Gordana Bogdavonic, Adnan Babovic, MizetaRizyano-vic, DzenitaLiuca et al. Cardiotocography in the Prog-nosis of Perinatal Outcome. Med Arch. 2014;68 (2): 102-5. DOI:10.5455/medarh.2014.68.102-105
16. Schifrin BS, Amsel J, Burd of G. The accuracy of auscultation detection of fetal cardiac deceleration: a computer simulation. Am J ObstetGnaecol. 1992;166:566-76. DOI:10.1016/0002-9378 (92) $91674-y$

17. Hafizur R, Renjhen P, Dutta S, Kar S. Admission cardiotocography: Itsrole in predicting foetal outcome inhigh-risk obstetric patients. AMJ. 2012; 5(10):522-527. doi: 10.4066/AMJ.2012.1267

18. Dr. Hrishikesh Joshi, Dr. Suruchi M Pawar, Dr. Akanksha Singh. Role of admission test by Cardiotocography (CTG) as a predictor of perinatal outcome: A prospective study. Int J ClinObstetGynaecol 2019;3(2):128-131. DOI:10.33545/gynae.2019.v3.i2c.21

19. Banu, S. : Relationship between Abnormal Cardiotocography and Fetal Outcome. Nepal Journal of Obstetrics and Gynaecology, 10(2), 36-39. Retrieved from https://www.nepjol.info/index.php/ NJOG/article/view/14334

20. Bhartiya V, Sharma R, Kumar A, Srivastava H. Admission Cardiotocography: A Predictor of Neonatal Outcome. J Obstet Gynaecol India. 2016 Oct;66(Suppl 1):321-9. DOI: 10.1007/s13224-016-0912-0. Epub 2016 Jun 14. PMID: 27651624; PMCID: PMC5016479 